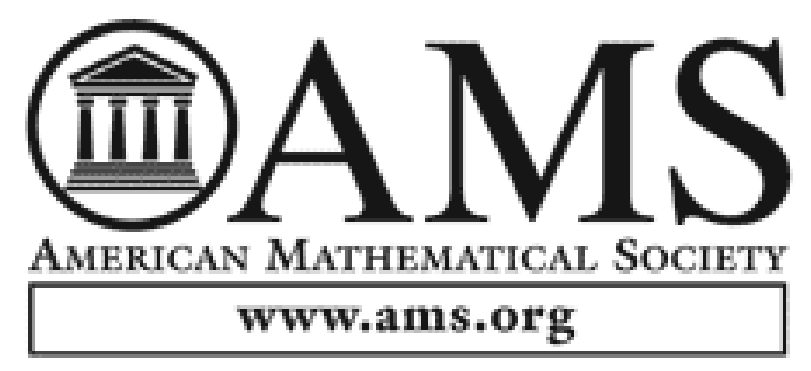

The Degree of Smooth Non-Arithmetically Cohen-Macaulay Threefolds in P5 Author(s): Rosa M. Miro-Roig

Source: Proceedings of the American Mathematical Society, Vol. 110, No. 2 (Oct., 1990), pp. 311-313

Published by: American Mathematical Society

Stable URL: http://www.jstor.org/stable/2048071

Accessed: 06/02/2009 07:04

Your use of the JSTOR archive indicates your acceptance of JSTOR's Terms and Conditions of Use, available at http://www.jstor.org/page/info/about/policies/terms.jsp. JSTOR's Terms and Conditions of Use provides, in part, that unless you have obtained prior permission, you may not download an entire issue of a journal or multiple copies of articles, and you may use content in the JSTOR archive only for your personal, non-commercial use.

Please contact the publisher regarding any further use of this work. Publisher contact information may be obtained at http://www.jstor.org/action/showPublisher?publisherCode=ams.

Each copy of any part of a JSTOR transmission must contain the same copyright notice that appears on the screen or printed page of such transmission.

JSTOR is a not-for-profit organization founded in 1995 to build trusted digital archives for scholarship. We work with the scholarly community to preserve their work and the materials they rely upon, and to build a common research platform that promotes the discovery and use of these resources. For more information about JSTOR, please contact support@jstor.org. 


\title{
THE DEGREE OF SMOOTH NON-ARITHMETICALLY COHEN-MACAULAY THREEFOLDS IN $\mathbf{P}^{5}$
}

\author{
ROSA M. MIRO-ROIG
}

(Communicated by Louis J. Ratliff, Jr.)

\begin{abstract}
In [B], Banica considers the problem of determining the integers $d$ such that there are smooth threefolds which are not arithmetically CohenMacaulay. Moreover, he gives a partial answer to this question. In this note, using liaison, we will complete his answer.
\end{abstract}

\section{INTRODUCTION}

In a recent work [B], Banica determines the integers $d$ such that there exist smooth surfaces of degree $d$ in $\mathbf{P}^{4}$ which are not arithmetically CohenMacaulay. Concretely, these are precisely the integers $d \geq 4$ with the exception $d=6$. Furthermore, he considers the problem of determining the integers $d$ such that there exist smooth threefolds in $\mathbf{P}^{5}$ which are not arithmetically Cohen-Macaulay, and he gives a partial answer to this question. Namely, for any odd integer $d \geq 7$ or any even integer $d=2 k>8$ with $k=5 s+1,5 s+2,5 s+3$ or $5 s+4$, there exist smooth threefolds in $\mathbf{P}^{5}$ of degree $d$ which are not arithmetically Cohen-Macaulay.

On the other hand, Beltrametti-Schneider-Sommese prove that any smooth threefold of degree 10 is arithmetically Cohen-Macaulay [BBS]. So, the problem of determining the integers $d=10 n, n>1$, such that there exist smooth threefolds in $\mathbf{P}^{5}$ which are not arithmetically Cohen-Macaulay, remains open.

The goal of this note is to prove that, for any integer $d=10 n, n>1$, there exist smooth threefolds in $\mathbf{P}^{5}$ of degree $d$ which are not arithmetically CohenMacaulay. To this end, we begin with well known smooth non-arithmetically Cohen-Macaulay threefolds in $\mathbf{P}^{5}$ of low degree, and we use the fact that the property of being arithmetically Cohen-Macaulay is preserved under liaison.

1. Let $\mathbf{k}$ be an algebraically closed field of characteristic zero, $S=\mathbf{k}\left[x_{0}, \ldots, x_{5}\right]$ and $\mathbf{P}^{5}=\operatorname{Proj}(S)$. Recall that a threefold $X$ in $\mathbf{P}^{5}$ is arithmetically CohenMacaulay if and only if $\oplus_{t \in \mathbf{Z}} H^{i}\left(\mathbf{P}^{5}, I_{X}(t)\right)=0$ for $1 \leq i \leq 3$. The notion of

Received by the editors September 26, 1989 and, in revised form, June 16, 1989.

1980 Mathematics Subject Classification (1985 Revision). Primary 14J30.

This research was partially supported by DGICYT PB88-0224. 
liaison among closed subschemes of $\mathbf{P}^{n}$ was introduced in [PS]; we will quote from that paper what we need in our proofs.

Our aim is to show

Proposition 1.1. For any integer $d=10 n, n>1$, there exist smooth threefolds in $\mathbf{P}^{5}$ of degree $d$ which are not arithmetically Cohen-Macaulay.

Proof. Let $Y \subset \mathbf{P}^{5}$ be a smooth non-arithmetically Cohen-Macaulay threefold of degree 12 having a locally free resolution of the following kind (see $[\mathrm{B}, \S 2.5]$ for the existence of $Y$ ):

$$
0 \rightarrow \mathscr{O} \oplus \mathscr{O}(1)^{3} \rightarrow \Omega(3) \rightarrow I_{Y}(6) \rightarrow 0 .
$$

In particular, $I_{Y}(6)$ is globally generated. Let $X$ be the threefold linked to $Y$ by means of two general hypersurfaces of degree 6 and 7, respectively, passing through $Y$. By [PS, Proposition 2.5], the ideal sheaf of $X$ has resolution

$$
0 \rightarrow T(-10) \rightarrow \mathscr{O}(-8)^{3} \oplus \mathscr{O}(-7)^{2} \oplus \mathscr{O}(-6) \rightarrow I_{X} \rightarrow 0
$$

In particular, the degree of $X$ is 30 , it is not arithmetically Cohen-Macaulay and $I_{X}(8)$ is globally generated. Now we use $X$ in order to construct nonarithmetically Cohen-Macaulay threefolds of degree $d=10 n, n \geq 5$.

In fact, for all $n \geq 5$, write $d+30=10(n+3)$ and take two general hypersurfaces of degree 10 and $n+3$, respectively, passing through $X$. As a residual, we get a smooth non-arithmetically Cohen-Macaulay threefold, $Z \subset$ $\mathbf{P}^{5}$, of degree $d=10 n, n \geq 5$.

Finally, it remains to construct smooth non-arithmetically Cohen-Macaulay threefolds of degree $d=20,40$.

Case $d=20$. Let $Y \subset \mathbf{P}^{5}$ be a smooth non-arithmetically Cohen-Macaulay threefold of degree 9 having a locally free resolution of the following kind (see $[\mathrm{B}, \S 2.5]$ for the existence of $Y$ ):

$$
0 \rightarrow T(-6) \rightarrow \mathscr{O}(-4)^{6} \rightarrow I_{Y} \rightarrow 0 .
$$

Note that $I_{Y}(4)$ is globally generated. So, taking two general hypersurfaces of degree 5 passing through $Y$, we get, as a residual, a smooth non-arithmetically Cohen-Macaulay threefold, $X \subset \mathbf{P}^{5}$, of degree 16. By [PS, Proposition 2.5], the ideal sheaf of $X$ has resolution

$$
0 \rightarrow \mathscr{O}(-6)^{6} \rightarrow \Omega(-4) \oplus \mathscr{O}(-5)^{2} \rightarrow I_{X} \rightarrow 0 .
$$

Finally, taking two general hypersurfaces of degree 6 passing through $X$ we get, as a residual, a smooth threefold of degree 20 , which is not arithmetically Cohen-Macaulay.

Case $d=40$. We take $Y$, a smooth non-arithmetically Cohen-Macaulay threefold of degree 9 as above, and two general hypersurfaces of degree 7 passing through $Y$. The residual threefold is smooth of degree 40 and it is not arithmetically Cohen-Macaulay. 
Corollary 1.2. For any integer $d \geq 7$ with exception $d=8,10$, there exist smooth threefolds in $\mathbf{P}^{5}$ which are not arithmetically Cohen-Macaulay.

Proof. It follows from [B], [BBS], and Proposition 1.1.

Remark 1.3. Until now there is no example of smooth subvariety of codimension 2 in $\mathbf{P}^{n}, n>5$, which is not arithmetically Cohen-Macaulay. Furthermore, Hartshorne conjectures that such an example does not exist $[\mathrm{H}]$.

\section{REFERENCES}

[B] C. Banica, Smooth reflexive sheaves, Preprint Series in Math. INCREST, Bucharest, Romanie 17 (1989), 1-43.

[H] R. Hartshorne, Varieties of small codimension in projective spaces, Bull. Amer. Math. Soc. 80 (1974), 1017-1032.

[BSS] M. Beltrametti, M. Schneider, and A. Sommese, Threefolds of degree 9 and 10 in $\mathbf{P}^{5}$, preprint, 1989.

[PS] C. Peskine and L. Szpiro, Liaison des varieties algebriques. I, Invent. Math. 26 (1974), 271-302.

Departamento de Algebra y Geometria, Universidad de Barcelona, Gran Viga 585 , 08007 Barcelona, Spain 